\title{
A RISK MEASURE ALTEIRATIVE TO THE VARIANCE
}

\author{
B. BerLiner \\ Zurich
}

\section{SUMMARY}

The qualifications of the semivariance as a useful risk measure are examined and compared to those of the variance. Although on first sight the semivariance may secm more appropriate from the insured's point of view the analysis of this paper Icack to a preference for the variance as a risk measure.

\section{INTRODUCTION}

Since the following considerations may be important for the reinsurance field the reacler can always replace the words "insurer" and "insured" by "reinsurer" and "reinsured". Regarding the variance as a risk measure for the insurer it is quite a natural question to ask whether the negative deviations

$$
x-E \text { with } x<E \text { and } E=\int_{-\infty}^{\infty} x d F(x)
$$

that are in favour of the insurer can or should be called risky. $F$ is the distribution function of the portfolio's total claims' amount.

If we answer our question with "no" a consequence woukl be to replace in the premium calculation for a portfolio the variance principle

$$
\pi=E+c V, c>o V=\sigma^{2}
$$

by a semivariance principle

$$
\pi=E+\bar{c} V_{+}, \quad \bar{c}>0
$$

where

$$
V^{\prime}=V_{+}+V_{-}=\int_{-\infty}^{\infty}(x-E)^{2} d l^{2}(x)
$$

and

$$
V_{+}=\int_{l:}^{\infty}(x-E)^{2} d F(x) ; V_{-}=\int_{-\infty}^{E}(x-E)^{2} d F(x)
$$


H. Markowitz, in his book "Portfolio Selection", chapter IX, I959 [10], comprehensively analyses the properties of the semivariance as a measure of variability in a portfolio analysis.

If the domain of definition of $F(x)$ is $[A, B]$ we can always define

$$
\tilde{F}(x)=\left\{\begin{array}{ll}
0 & \text { for }-\infty<x<A \\
F(x) & \text { for } A \leq x \leq B \\
I & \text { for } B<x<\infty
\end{array} \text { and replace } F(x) \text { by } l^{\prime}(x)\right.
$$

The terms $c V$ in (I), $\bar{c} V+$ in (2) respectively are meant to be pure risk loadings. Loadings for administrative costs, commissions, etc. are not considered.

The purpose of this paper is to investigate whether the variance principle $\pi=E+c V$ should be replaced by the semivariance principle $\pi=E+\bar{c} V+$.

The lower integral limit in (3) shows that we also allow for negative losses which can for example occur when due to a juclgement of a court of appeal the insured has to repay the insurer part of the payments that he received in previous years.

The possible use of $V_{+}$has already been mentioned or cven recommended sevcral times [2], [3], [6].

A. Properties of $V_{+}$

AI. $\quad V_{+}$depends only on the expected value $E$ of the distribution function $F(x)$ and on the structure of $F(x)$ for $x \geq E$.

A2. $V+\leq$ and $V_{+}=V<=>V=0$.

Therefore, if we replace in a premium calculation a $V$-loading by a $V+$-loading we should enlarge the loading's coefficient.

A3. For $\int_{-\infty}^{\infty} x f(x) d x=\int_{-\infty}^{\infty} x g(x) d x=E$ and $f \leq g$ for $x \geq E$ follows $V_{+f} \leq V_{+}$.

A4. Let us assume one point of intersection $x>E$ and let $f(\bar{x})=g(\bar{x}), f(x)>g(x)$ for $E \leq x<\bar{x}$ and $g(x)>f(x)$ for $x>\bar{x}$.

Let us moreover assume

$$
\begin{aligned}
& \int_{E}^{\bar{x}}(x-E)^{1+n}(f(x)-g(x)) d x \leq \\
& \leq \int_{i}^{\infty}(x-E)^{1+n}(g(x)-f(x)) d x \text { with }-\mathrm{I} \leq \eta<\mathrm{I} .
\end{aligned}
$$


Assertion: Then follows $V_{+f}<V_{+g}$.

Proof:

$$
\begin{aligned}
\int_{E}^{\bar{x}}(x-E)^{2} & (f(x)-g(x)) d x<(\bar{x}-E)^{1-n} \int_{B}^{\bar{x}}(x-E)^{1+n}(f(x)-g(x)) d x \\
& \leq(\bar{x}-E)^{1-n} \int_{\bar{x}}^{\infty}(x-E)^{1+n}(g(x)-f(x)) d x< \\
& <\int_{\bar{z}}^{\infty}(x-E)^{2}(g(x)-f(x)) d x \\
& =>\int_{\bar{z}}^{\infty}(x-E)^{2}(g(x)-f(x)) d x- \\
& -\int_{E}^{\bar{z}}(x-E)^{2}(f(x)-g(x)) d x= \\
& =\int_{B}^{\infty}(x-E)^{2}(g(x)-f(x)) d x=V_{+g}-V_{+f}>0 \quad \text { c.e.d. }
\end{aligned}
$$

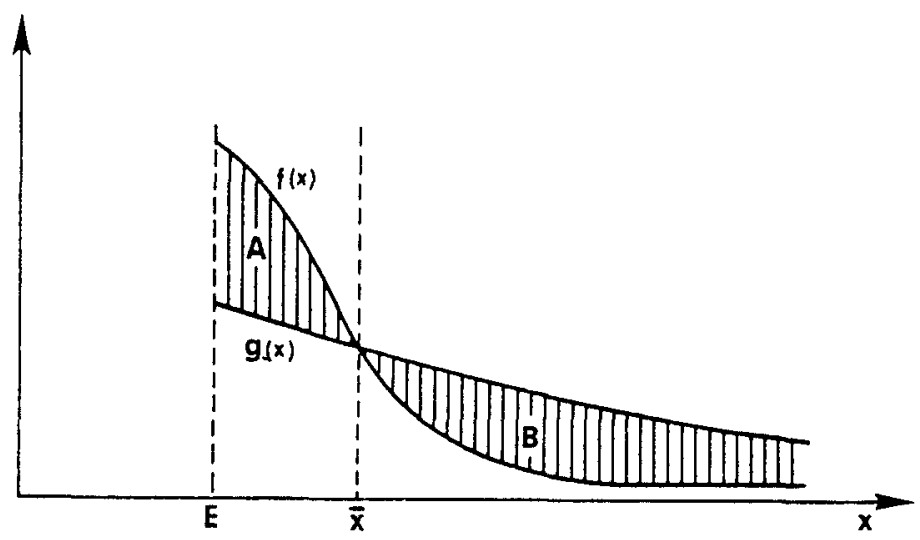

lig. I

Corrolary I: For $\eta=-I$ we arrive at $\Lambda=\int_{E}^{\bar{x}}(f(x)-g(x)) d x \leq \int_{\bar{x}}^{\infty}(g(x)-f(x)) d x=B($ Figr. I $)=>V_{+f}<V_{+g}$

Corrolary $2:$ For $\eta=0$ we arrive at:

$\hat{E}_{+\mathrm{I}}=\int_{E}^{\infty}(x-E) g(x) d x \geq \int_{E}^{\infty}(x-E) f(x) d x=\hat{E}_{+\mathrm{II}}=>V_{+\mathrm{I}}>V_{+\mathrm{II}}$ 
i.e. if two portfolios I and II-characterized by the distribution densities $g(x)$ and $f(x)$ (Fig. I), both of which have the same pure loss cost $E$-would have pure stop loss premiums $\hat{E}_{+\mathrm{I}}>\hat{E}_{+\mathrm{II}}$ excess $E$ then follows for the respective loadings $V_{+\mathrm{I}}>V_{+\mathrm{II}}$.

The questions arisc here firstly whether we should not usc the pure stop loss premium excess the expected valuc

$$
\hat{E}_{+}=\int_{n}^{\infty}(x-E) d F(x),
$$

as an alternative to the standard deviation loading (dealt with in [6]) and secondly what its relations to $V_{+}$(except for the alrcady above-mentioned corollary 2) are.

Approximations and an upper limit for $\hat{E}_{+}$are given in [3] and [8].

A5. From A4. and Fig. I we can follow that $V_{+}$is the larger, the farther away to the right of $E$ are substantial probabilities of claims occurrences.

We could therefore believe at the first moment that $V+$ is closely connected to the third central moment $\mu_{3}$ which, to a certain degree, characterizes the dangerousness of a distribution function or of a portfolio.

The argument often used is that given two risks or portfolios having the same first and second central moments $E, \sigma^{2}$, the one with the larger third central moment $\mu_{3}$ or skewness $\gamma$ is the less desirable one for the insurer becausc it is more dangerous [2], [9]. (J. Marschak for example proposed the use of the skewness $\gamma$ as a risk measure already in $\mathrm{I}_{93} 8$ [II].) The above argument is certainly correct for most distribution functions used in and needed for insurance. Let us, however, imagine a distribution function with "enough" parameters which we could change in such a way that $E, \sigma^{2}$ and the distribution functions for $x>E$ remain unchanged while we are diminishing $\mu_{3}$ perpetually by enlarging the potential amounts of substantial profits with substantial but diminishing probabilitics (deviations to the left from the expected value) (Fig. 2).

$$
E_{I}=E_{I I}, V_{I}=V_{I I}, V_{+I}=V_{+I I}, \mu_{3 I}>\mu_{3 I I}, \gamma_{I}>\gamma_{I I} .
$$

Would an insurance company say that portfolio $I$ is more dangerous than portfolio II and thus prefer portfolio II to portfolio I? 
Can we at all speak of dangerousness when referring only to amounts of profit? We think not, especially when the company utilizes the profit $z=\pi-x$ with a function $u(z)$ with $u^{\prime}(z)>0$ and $u^{\prime \prime}(z)<0$ such that $E_{\mathrm{I}}[u(z \mid z>\pi-E)]>E_{\mathrm{II}}[u(z \mid z>\pi-E)]$, thus making portfolio II more "dangerous" respectively less profitable than portfolio I.

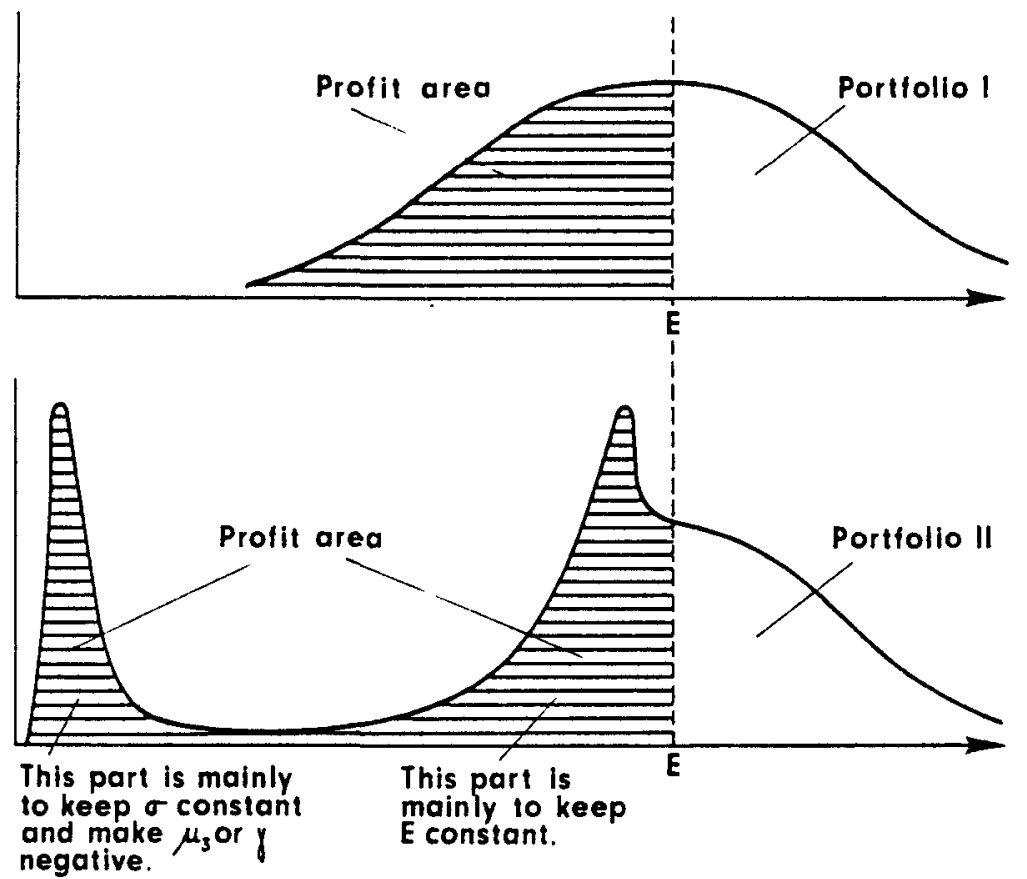

Fig. 2

Finally we can follow from the above written and Fig. 2 that from $V+$ I $\leq V_{+}$II does not follow $\mu_{3 I} \leq \mu_{3 I I}$ or

$$
\text { from } \frac{V_{+I}}{V_{I}} \leq \frac{V_{+I I}}{V_{I I}} \text { does not follow } \gamma_{I} \leq \gamma_{I I}
$$

and vice versa.

The above reflections and those made in the introduction lead us to the conclusion that $V_{+}$is. a better risk measure with respect to the content of the word "dangerousness", than $V$ or $\mu_{3}$ or the linear combination $c V+d \mu_{3}$. 
B. Numerical examples to illustrate some properties, in particular property A5.

\begin{tabular}{cccccc}
\hline \multicolumn{5}{c}{ Density functions describing the probabilitics of claims $x$} \\
\hline$x$ & $f^{(1)} x$ & $f^{(2)}(x)$ & $f^{(3)}(x)$ & $f^{(4)}(x)$ & $f^{(3)}(x)$ \\
\hline-10 & 0 & 0 & 0 & 0,0001 & 0,02 \\
0 & 0,18 & 0,198 & 0,076 & 0,0529 & 0,40 \\
0,5 & 0 & 0 & 0 & 0,0440 & 0 \\
$I$ & 0,80 & 0,800 & 0,920 & 0,8990 & 0 \\
2 & 0 & 0 & 0 & 0 & 0,50 \\
10 & 0,02 & 0 & 0 & 0 & 0,02 \\
20 & 0 & 0 & 0,004 & 0,0040 & 0 \\
100 & 0 & 0,002 & 0 & 0 & 0 \\
\hline$I^{2}$ & 1,00 & 1,000 & 1,000 & 1,0000 & 1,00 \\
$V$ & 1,80 & 19,800 & 1,520 & 1,5200 & 5,00 \\
$I^{\prime}+$ & 1,62 & 19,002 & 1,444 & 1,4440 & 2,12 \\
$\mu_{3}$ & $I 4,40$ & 1940,400 & 27,436 & 27,2445 & $-12,00$ \\
\hline
\end{tabular}

All the above distribution functions have the same expected value $E=\mathrm{I}$.

BI. The density function $f^{(2)}(x)$ illustrates as compared to $f^{(1)}(x)$ numerically the fact that if the function's "tail" grows linearly and the "tail's" probability diminishes linearly, $V$ and $V+$ are growing "almost linearly", $\mu_{3}$ "almost quadratically". $E$ does not change here at all, usually only "a little bit".

This shows how very dangerous it can be to use risk loadings of third and larger order if the portfolio includes very large or even catastrophe risks with an unknown, small probability of occurrence.

B2. Since $V_{+}^{(1)}>V_{+}^{(3)}$ we would conclude according $t_{0}$ property $\mathrm{A} 5$. that risk I which is described by $f^{(1)}(x)$ is more dangerous than risk III which is described by $f^{(3)}(x)$, although $\mu_{3}^{(1)}<\mu_{3}^{(3)}$

Let us imagine an insurance company with a utility function $u(\pi-x)=u(z)=5\left(\mathrm{I}-c^{-2 / 5}\right)$ that can get a premium of $\pi=I, 4$ for insuring either risk I or risk III. The calculation of the respective expected utilities lead to 


$$
\begin{array}{lr}
E_{\mathrm{I}}(u(z))= & 0,07 \\
E_{\text {III }}(u(z))= & -0,36 .
\end{array}
$$

Thus the insurance company would prefer to insure risk I rather than risk III. It considers risk III more dangerous and less attractive than risk $\mathrm{I}$.

We constructed $f^{(3)}(x)$ to show that $V_{+}$also is assailable with respect to its reflecting "dangerousness", however, this is true for cvery risk measure.

We nevertheless prefer the result given in A5., i.c. $V_{+}$to $V$ or $\mu_{3}$ as a measure of dangerousness.

B3. The density function $f^{(4)}$ has been constructed in comparison to $f^{(3)}$ according to the procecding described in $\mathrm{A}_{5}$.

$$
\begin{aligned}
E^{(4)} & =E^{(3)} \\
V^{(4)} & =V^{(3)} \\
V_{+}^{(4)} & =V_{+}^{(3)} \\
f_{(4)}^{(x)} & =f_{(3)}^{(x)} \text { for } x>E^{(3)}=E^{(4)}=\mathrm{I} \\
\text { and } \quad \mu_{3}^{(4)} & <\mu_{3}^{(3)}
\end{aligned}
$$

B34. The density function $f^{(5)}$ having a negative third central moment shows more significantly than $f^{(4)}$ the contrast to $f^{(3)}$ and the fact that

$$
V_{+\mathrm{I}} \leq V_{+\mathrm{II}}<\#>\mu_{3 \mathrm{I}} \leq \mu_{3 \mathrm{II}}
$$

Thus $E^{(3)}=E^{(1)}=E^{(5)}$

$$
\begin{aligned}
& V^{(3)}<V^{(1)}<V^{(5)} \\
& V^{(3)}<V_{+}^{(1)}<V_{+}^{(5)} \\
& \mu_{3}^{(3)}>\mu_{3}^{(1)}>0>\mu_{3}^{(5)} .
\end{aligned}
$$

C. Explicit expressions for $V_{+}$and $V_{+} / V$ for some distribution functions that are of special imporlance in insurance and reinsurance

Cr. Normal distribution

$$
\begin{gathered}
f(x)=\frac{I}{\sqrt{2 \pi} \sigma} e^{-\frac{(x-y)^{2}}{2 \pi^{2}}},-\infty<x<\infty,-\infty<\mu<\infty, \quad 0<\sigma<\infty \\
E=\mu ; V^{\prime}=\sigma^{2} ; V+=\frac{1}{2} \sigma^{2} ; V+V=\frac{1}{2}
\end{gathered}
$$


C2. Since the classical approximation of the generalized Poisson distribution function is the normal distribution function [I], we arrive for this approximation (first term of Edgeworth expansion) at the same result as in $\mathrm{C}_{\text {I. }}$.

$C_{3}$. For every symmetrical distribution function we have

$$
V_{+} / V=\frac{1}{2} \text {. }
$$

C4. Exponential distribution

$$
\begin{aligned}
& f(x)=c e^{-c x} 0<x<\infty, 0<c<\infty \\
& E=\mathrm{I} / c ; V=\mathrm{I} / c^{2} ; V_{+}=2 / c \cdot \mathrm{I} / c^{2} ; V_{+} / V=2 / e .
\end{aligned}
$$

C5. Gamma distribution

$$
\begin{aligned}
& f(x)=\frac{c^{\gamma}}{\Gamma(\gamma)} e^{-c x} x^{\gamma-1} 0<x<\infty, 0<\gamma<\infty, 0<c<\infty \\
& E=\frac{\gamma}{c} ; V=\frac{\gamma}{c^{2}} ; V_{+}=\frac{I}{c^{2} \Gamma(\gamma)}\left\{e^{-\gamma} \gamma^{\gamma}+\Gamma(\gamma+I)-\gamma_{\gamma}^{\Gamma}(\gamma)\right\} ; \\
& \frac{V_{+}}{V}=\frac{I}{\Gamma(\gamma)}\left\{e^{-\gamma} \gamma^{\gamma-1}+\Gamma(\gamma)-\Gamma_{\gamma}(\gamma)\right\} .
\end{aligned}
$$

Thus $V_{+} / V$ depends only on $\gamma$, not on $c$. In the special case of $\gamma=I$ we arrive at the exponential case that was discussed in $\mathrm{C}_{4}$.

$\begin{array}{cc}\gamma & V+/ V \\ 0,5 & 0,80 I \\ I & 0,736 \\ 2 & 0,677 \\ 3 & 0,647 \\ 4 & 0,629 \\ 5 & 0,6 \mathrm{I} 6 \\ 6 & 0,606\end{array}$

For large $\gamma$ we can use the Stirling formula:

I.et us define $\eta=\gamma-\mathrm{I}$

$$
\begin{aligned}
\Gamma(\gamma)=\Gamma(\eta+I) \sim \eta^{i} e^{-\eta} & \int_{0}^{\infty} e^{-1 / 2 \eta(x-n)^{2}} d x=\eta^{n+1 / 2} e^{-\eta} \int_{-\gamma \cdot \bar{n}}^{\infty} e^{-1 / 2 t^{2}} d t \\
& \sim \eta^{+1 / 2} e^{-\eta} \int_{-\infty}^{\infty} e^{-1 / 2 t^{2}} d t=\sqrt{2 \pi} \eta^{\eta+1 / 2} e^{-n}[I 2]
\end{aligned}
$$




$$
\begin{array}{r}
\Gamma(\gamma)-\Gamma_{\gamma}(\gamma)=\Gamma(\eta+I)-\Gamma_{\eta+1}(\eta+I)=\int_{\eta+1}^{\infty} z^{\eta} e^{-z} d z \sim \eta^{\eta+1 / 2} e^{-\eta} \int_{1 / V \bar{\pi}}^{\infty} e^{-1 / 2 t^{2}} d t \\
\sim \frac{1}{2} \sqrt{2 \pi} \eta^{\eta+1 / 2} e^{-\eta} \sim \frac{1}{2} \Gamma(\gamma) .
\end{array}
$$

Thus

$$
\begin{aligned}
\lim _{\gamma \rightarrow \infty} \frac{V_{+}}{V} & =\lim _{\gamma \rightarrow \infty} \frac{e^{-\gamma} \gamma^{\gamma-1}}{\sqrt{2 \pi} c^{-(\gamma-1)}(\gamma-I)^{\gamma-1 / 2}}+\lim _{\gamma \rightarrow \infty} \frac{\Gamma(\gamma)-\Gamma_{\gamma}(\gamma)}{\Gamma(\gamma)} \\
& =\lim _{\gamma \rightarrow \infty} \frac{I}{\sqrt{2 \pi} c}\left(\frac{\gamma}{\gamma-I}\right)^{\gamma-1 / 2} \cdot \frac{I}{\sqrt{\gamma}}+\frac{1}{2} \\
& =\lim _{\gamma \rightarrow \infty} \frac{I}{\sqrt{2 \pi \gamma}}+\frac{1}{2} \\
\lim _{\gamma \rightarrow \infty} \frac{V+}{V} & =\frac{1}{2}
\end{aligned}
$$

We want to calculate now the other extreme, namely $\lim _{\gamma \rightarrow 0} V_{+} / V$

$$
\begin{gathered}
\lim _{\gamma \rightarrow 0} \Gamma(\mathrm{I}+\gamma)=\Gamma(\mathrm{I})=\mathrm{I} \\
\lim _{\gamma \rightarrow 0} \frac{V_{+}}{V}=\lim _{\gamma \rightarrow 0}\left(\mathrm{I}+\gamma^{\gamma} e^{-\gamma}-\gamma \int_{0}^{\gamma} e^{-z} z^{\gamma-1} d z\right)= \\
=\mathrm{I}-\lim _{\gamma \rightarrow 0}\left(\int_{0}^{\gamma} e^{-z} z^{\gamma} d z\right)
\end{gathered}
$$

For $0<\gamma<$ I we have

$$
0<\int_{0}^{\gamma} e^{-z} z^{\gamma} d z<\int_{0}^{\gamma} e^{-z} d z=\mathrm{I}-c^{-\gamma}=\gamma-\frac{\gamma^{2}}{2 !}+-\ldots
$$

Thus

$$
\begin{gathered}
\lim _{\gamma \rightarrow 0}\left(\int_{0}^{\gamma} e^{-z} z^{\gamma} d z\right)=0 \text { and } \\
\lim _{\underline{\gamma \rightarrow 0}} \frac{V+}{V}=I .
\end{gathered}
$$

As we see $V / V_{+}$is independent of $c$ and is only slowly decreasing as a function of $\gamma$, slowly especially for $\gamma \geq 2$.

C6. Pareto distribution

This distribution is of special importance for the excess of loss reinsurer. 


$$
\begin{gathered}
f(x)=\alpha a^{\alpha} x^{-\alpha-1} a \leq x<\infty, I<\alpha<\infty, 0<a<\infty \\
E=a \frac{\alpha}{\alpha-I} ; V=\frac{a^{2} \alpha}{(\alpha-I)^{2}(\alpha-2)} ; V_{+}=\frac{2 a^{2}}{(\alpha-I)(\alpha-2)}\left(\frac{\alpha-I}{\alpha}\right)^{\alpha-2} \\
\frac{V+}{V}=2\left(\frac{\alpha-I}{\alpha}\right)^{\alpha-1}=2\left(\frac{\alpha}{\alpha-I}\right)^{-\alpha+1}
\end{gathered}
$$

While $V$ and $V+$ exist only for $\alpha>2 V+/ V$ like $E$ exists for $\alpha>I$, though for $\mathrm{I}<\alpha<2, V_{+} / V>\mathrm{r}$ and thus does not makes sense.

$$
\begin{aligned}
& \propto \quad V+/ V \\
& 2 \text { I } \\
& 2,5 \quad 0,930 \\
& 3 \quad 0,889 \\
& 4 \quad 0,844 \\
& 5 \quad 0,819 \\
& \text { I0 } \quad 0,775 \\
& \underline{\frac{d}{d \alpha}}\left(\frac{V_{+}}{V}\right)=-2\left(\sum_{i=2}^{\infty} \frac{\mathrm{I}}{i \alpha^{i}}\right) \cdot\left(\frac{\alpha-\mathrm{I}}{\alpha}\right)^{\alpha-1}<0 \\
& \lim _{\alpha \rightarrow 2} \frac{V+}{V}=\lim _{\alpha \rightarrow 2} 2\left(\frac{\alpha-I}{\alpha}\right)^{\alpha-1}=I \\
& \lim _{\alpha \rightarrow \infty} \frac{V+}{V}=2 \lim _{\alpha \rightarrow \infty}\left(I-\frac{I}{\alpha}\right)^{\alpha-1}=\frac{2}{e}
\end{aligned}
$$

The quotient $V / V_{+}$is independent of $a$ and a slowly decreasing function of $\alpha$.

C7. Log normal distribution

$$
\begin{gathered}
f(x)=\frac{I}{\sqrt{2 \pi} \sigma x} e^{-\frac{(\ln x-\mu)^{2}}{2 \sigma^{2}}} 0 \leq x<\infty,-\infty<\mu<\infty, 0<\sigma<\infty \\
E=e^{\mu+\sigma^{2} / 2} ; V=e^{2 \mu+\sigma^{2}}\left(e^{\sigma^{2}}-\mathrm{I}\right) \\
V+=\int_{E}^{\infty}(x-E)^{2} \frac{I}{\sqrt{2 \pi} \sigma x} e^{-1 / 2\left(\frac{\ln x-\mu}{\sigma}\right)^{2}} d x \\
=\frac{I}{\sqrt{2 \pi}} \int_{\sigma / s}^{\infty}\left(e^{2 \sigma z+2 \mu}-2 e^{\sigma^{2} / 2+\sigma z+2 \mu}+e^{\sigma^{2}+2 \mu}\right) e^{-1 / 2 z^{2}} d z
\end{gathered}
$$


wherc

$$
z=\frac{\ln x-\mu}{\sigma} .
$$

Denoting $\phi(z)=I / \sqrt{2 \pi} \int_{-\infty}^{2} e^{-u^{2} / 2}$ du we arrive after some simple calculations and substitutions at the expression:

$V_{+}=E^{2}\left\{e^{\sigma^{2}}\left(\mathrm{I}-\phi\left(-\frac{3}{2} \sigma\right)\right)-\left(\mathrm{I}-\phi\left(-\frac{\sigma}{2}\right)\right)-\left(\phi\left(\frac{\sigma}{2}\right)-\phi\left(-\frac{\sigma}{2}\right)\right)\right\}$

Thus

$$
\frac{\frac{V_{+}}{V}=\frac{\mathrm{I}}{\left(e^{\sigma^{2}}-\mathrm{I}\right)}\left\{e^{\sigma^{2}}\left(\mathrm{I}-\phi\left(-\frac{3}{2} \sigma\right)\right)-\left(\mathrm{I}-\phi\left(-\frac{\sigma}{2}\right)\right)-\left(\phi\left(\frac{\sigma}{2}\right)-\right.\right.}{\left.\left.-\phi\left(-\frac{\sigma}{2}\right)\right)\right\}}
$$$$
\lim _{\sigma \rightarrow \infty} \frac{V_{+}}{V}=\lim _{\sigma \rightarrow \infty} \frac{e^{\sigma^{2}}}{e^{\sigma^{2}}-I} \cdot \frac{I}{\sqrt{2 \pi}} \int_{-0 / 20}^{\infty} e^{-1 / 2 t^{2}} d t-
$$$$
-\lim _{n \rightarrow \infty}-\frac{I}{e^{\sigma^{2}}-I} \frac{I}{\sqrt{2 \pi}} \int_{-\sigma / 2}^{\infty} e^{-1 / 2 t^{2}} d t
$$$$
-\lim _{\sigma \rightarrow \infty} \frac{I}{e^{\sigma^{2}}-I} \frac{I}{\sqrt{2 \pi}} \int_{-\sigma / 2}^{\sigma / 2} e^{-1 / 2 t^{2}} d t=I-0-0=I
$$

$\lim _{\sigma \rightarrow 0} \frac{V}{V_{+}}=\lim _{\sigma \rightarrow 0} \frac{I}{\sigma^{2}+o\left(\sigma^{4}\right)}\left(\left(I+\sigma^{2}+o\left(\sigma^{4}\right)\right)\left(\frac{I}{2}+\frac{I}{\sqrt{2 \pi}} \cdot \frac{3}{2} \sigma+o\left(\sigma^{3}\right)\right)\right.$

$$
\begin{aligned}
& -\frac{I}{2}-\frac{I}{\sqrt{2 \pi}} \frac{\sigma}{2}-\frac{2}{\sqrt{2 \pi}} \cdot \frac{\sigma}{2} \\
& =\lim _{\sigma \rightarrow 0} \frac{I / 2 \sigma^{2}+o\left(\sigma^{3}\right)}{\sigma^{2}+o\left(\sigma^{4}\right)}=\frac{I}{2} .
\end{aligned}
$$


The quotient $V / V_{+}$is independent of $\mu$ and a slowly increasing function of $\sigma$, slowly increasing especially for $\sigma \geq \mathrm{I}$.

C8. I.ct the portfolio's claims function be generated by a Poisson process (parameter $\lambda$ ) with all claims being of equal sizes.

$$
\begin{aligned}
& P(X=n s)=e^{-\lambda} \frac{\lambda^{n}}{n !}, \quad 0<\lambda<\infty, 0<s<\infty \\
& E=\lambda \cdot s ; V=\lambda \cdot s^{2} \\
& V_{+}=\int_{E}^{\infty}(x-E)^{2} d F(x)=V-\int_{0}^{E}(E-x)^{2} d F^{F}(x)= \\
& =\lambda s^{2}\left(\mathrm{I}-\lambda \cdot \sum_{n=0}^{[\lambda j}\left(\mathrm{I}-\frac{n}{\lambda}\right)^{2} c^{-\lambda} \frac{\lambda^{n}}{n !}\right) \\
& =\lambda s^{2}\left(I-\lambda \sum_{n=0}^{[\lambda]} e^{-\lambda} \frac{\lambda^{n}}{n !}+2 \lambda \sum_{n=0}^{[\lambda-1]} e^{-\lambda} \frac{\lambda^{n}}{n !}-\lambda \sum_{n=0}^{[\lambda-2]} e^{-\lambda} \cdot \frac{\lambda^{n}}{n !}-\right. \\
& \left.-\sum_{n=1}^{[\lambda-1]} c^{-\lambda} \frac{\lambda^{n}}{n !}\right) \\
& =\lambda s^{2} \cdot e^{-\lambda}\left(\frac{\lambda^{[2]}}{[\lambda-I] !}-\frac{\lambda^{[\lambda+1]}}{[\lambda] !}+\sum_{n=[\lambda]}^{\infty} \frac{\lambda^{n}}{n !}\right) \\
& \frac{V_{+}}{V}=e^{-\lambda}\left(-\frac{\lambda^{[\lambda]}}{[\lambda] !}(\lambda-[\lambda])+\sum_{n=[\lambda]}^{\infty} \frac{\lambda^{n}}{n !}\right)
\end{aligned}
$$

where $[\lambda]$ is the integer part of $\lambda$.

If $\lambda$ is an integer, i.e. $\lambda=[\lambda]$ then we have

$$
\begin{array}{cc}
\frac{V_{+}}{V}=e^{-\lambda} \sum_{n-\lambda}^{\infty} \frac{\lambda^{n}}{n !}= & \mathrm{I}-e^{-\lambda} \sum_{n=0}^{\lambda-1} \frac{\lambda n}{n !} \\
\lambda & V_{+} / V \\
I & 0,632 \mathrm{I} \\
2 & 0,5940 \\
5 & 0,5595 \\
\text { I0 } & 0,542 \mathrm{I}
\end{array}
$$




$$
\begin{aligned}
\underline{\lim _{\lambda \rightarrow 0} \frac{V_{+}}{V}=\lim _{\lambda \rightarrow 0} e^{-\lambda}\left[\sum_{n=0}^{\infty} \frac{\lambda^{n}}{n !}-\lambda\right]=\mathrm{I}-\lim _{\lambda \rightarrow 0} \lambda e^{-\lambda}=\mathrm{I}} \\
\lim _{\lambda \rightarrow \infty} \frac{V_{+}}{V}=-\lim _{\lambda \rightarrow \infty} \frac{\lambda^{[\lambda]}}{[\lambda] !}(\lambda-[\lambda]) e^{-\lambda}+\mathrm{I}-\lim _{\lambda \rightarrow \infty} \sum_{n=0}^{[\lambda-1]} e^{-\lambda} \frac{\lambda^{n}}{n !} \\
=\mathrm{I}-\lim _{\lambda \rightarrow \infty} \sum_{n=0}^{[\lambda]} e^{-\lambda} \frac{\lambda^{n}}{n !}+\lim _{\lambda \rightarrow \infty}(\mathrm{I}+[\lambda]-\lambda) \frac{\lambda^{[\lambda]}}{[\lambda] !} e^{-\lambda} \\
=\mathrm{I}-\lim _{\lambda \rightarrow \infty} \int_{0}^{\lambda} \frac{\mathrm{I}}{\sqrt{2 \pi \lambda}} e^{-\frac{(z-\lambda)^{2}}{2 \lambda}}\left(\mathrm{I}+0\left(\frac{\mathrm{I}}{\lambda}\right)\right) d z+0 \\
=\mathrm{I}-\lim _{\lambda \rightarrow \infty} \int_{-\infty}^{\lambda} \frac{\mathrm{I}}{\sqrt{2 \pi \lambda}} e^{-\frac{(z-\lambda)^{2}}{2 \lambda}} d z= \\
=\mathrm{I}-\lim _{\lambda \rightarrow \infty} \int_{-\infty}^{\infty} \frac{\mathrm{I}}{\sqrt{2 \pi \lambda}} e^{-\frac{(z-\lambda)^{2}}{2 \lambda}} d z=\frac{1}{2} \\
\frac{\lim _{\lambda \rightarrow \infty} \frac{V}{V+}}{\underline{V}}=\frac{1}{2} .
\end{aligned}
$$

The quotient $V_{+} / V$ is independent of $s$ and a slowly decreasing function of $\lambda$, slowly decreasing especially for $\lambda \geq I$.

\section{Remark I:}

In cases $\mathrm{CI}_{\mathrm{I}}-\mathrm{C}_{4} . V_{+} / V$ is a fixed number, whereas in cases $\mathrm{C}_{5}-\mathrm{C} 8$. $V_{+} / V$. depends only on one parameter and is only slowly varying with that parameter, especially in the parameter regions that are interesting for the insurer.

\section{Remark 2:}

In all the cases CI.-C8. is $V_{+} / V \geq \frac{1}{2}$ and $\mu_{3} \geq 0$ (for Pareto $\mu_{0}$ exists only for $\alpha>3)$. Also in the numerical examples we have for $f^{(1)}(x), f^{(2)}(x), f^{(3)}(x), f^{(4)}(x) V_{+} / V>\frac{1}{2}, \mu_{3}>0$ whereas for $f^{(5)}(x)$ we observe $V_{+} / V<\frac{1}{2}$ and $\mu_{3}<0$.

The question arises therefore if the hypothesis $V_{+} \mid V \geq \frac{1}{2}<=>$ 
$\mu_{3} \geq 0$ is correct. This is not the case as we can conclude from the following counter example.

Example.

Let $f(x)=\left\{\begin{array}{lr}0,02857 \text { for } x= & -7 \\ 0,45143 \text { for } x= & 0 \\ 0,50000 \text { for } x= & 2 \\ 0,02000 \text { for } x= & \text { I0 }\end{array}\right.$

$E=1,000 ; \quad V=4,400 ; \quad V_{+}=2,120 ; \quad V_{+} / V=0,482<0,500 ;$

$\mu_{3}=0,00073>0$.

D. Is $V+$ to be preferred in general to $V$ as a risk measure or a risk loading?

When analyzing $V$ and $V+$ from a portfolio selection point of view and putting up pros and cons, $\mathrm{H}$. Markowitz does not come to a universal proposal as to which of the two risk measures is to be preferred [Io].

For all that, Markowitz writes in [IO] on page 194: "Analyses based on $S$ (our $V_{+}$) tend to produce better portfolios than those based on $V . "$

The main difference between an analysis considering appropriate risk measures for the calculation of a promium or for a porlfolio selcction are the underlying conditions and constraints. While we may wish in insurance to establish a premium principle that takes the dangerousness of a portfolio and/or the lack of statistics into account, that is as just as possible to all customers that automatically sets up an upper barrier of acceptance and so on, we may for example wish to select a shares- or bonds-portfolio in such a way that to a given expected return for the invested capital $V$ or $V_{+}$becomes a minimum.

The choice between $V$ or $V+$ may therefore fall out differently for a portfolio selection principle and for a premium calculation principle. We are interested here in the latter case.

Having shown until now advantageous properties of $V+$ we would like to list some disadvantageous properties in comparison to $V$ or the lack of properties of $V_{+}$that we would like a risk measure to have and that $V$ possesses. 
DI. The variance loading is additive, i.c. the loading assigned to the sum of two independent risks is the sum of the loadings that are assigned to the two risks independently.

On the other hand the semivariance loading does not possess the property of additivity.

D2. Usually $V_{+}$is more difficult and more time-consuming to calculate than $V$.

If we wish to calculate for example the premium of a portfolio consisting of $n$ independent risks each of which has a distribution function $F_{1}(x), \ldots \ldots, F_{n}(x)$ then we need, in case of a $V$-loading, to calculate only the variances of every risk and add them up. In case of a $V$-loading the convoluted joint distribution function $F_{1}(x) * F_{2}(x) * \ldots \ldots * F_{n}(x)$ has to be determined for the calculation of $V+$ which is usually complicated and time-consuming.

D3. For example if $F_{1}(x), \ldots \ldots, F_{n}(x)$ are Pareto distributions their convolution can not be written as a closed analytical expression. Thus there exist cases when $V$ can be calculated easily and exactly and $V+$ can not be calculated exactly at all and an approximation can only be got after complicated calculations.

D4. For a large class of infinitely divisible functions we arrive in a first approximation at a variance loading if a company adds an independent marginal treaty to its portfolio, without changing its probability of obtaining a ncgative result [7], [4].

We have here an important property that characterizes a $V$ loading and that a $V_{+}$-loading does not possess.

Not changing the probability of obtaining a negative result means taking into consideration all possible results, losses as well as profits. From this point of view it is logical to include the possible profitable results in the risk measure which is done when using $V$, but not when using $V_{+}$as a risk measure.

If an insurance company considers its internal problems and does not want to increase its probability of ruin or of loss over a certain period of time, i.e. if it looks upon a risk subjectively and not objectively, its contemplations should lead it to a variance rather than to a semivariance loading. 
D5. Because of their quadratic nature the variance as well as the semivariance loadings lead to an equilibrium state in an insurance market. For each cover there exists a price minimum and belonging to it fixed shares of the cover for each insurer and reinsurer in the market. For the variance loading a simple and useful approximation leads to shares that are very easily calculable [5].

Such a simple and useful solution to the equilibrium problem is not known and probably does not exist if the semivariance is used as a risk measure.

\section{E. Final conclusion}

In all cases dealt with in $\mathrm{C}_{1}$-C8. all of which are important for insurance $V_{+} / V$ depended on less parameters than the underlying distribution function and was either constant or dependent on one parameter only.

In all cases where $V_{+}=$constant $\cdot V$ the variance and semivariance principles were identical since we can write

$$
\pi=E+\bar{c} V=E+c V \text { with } c=\bar{c} \cdot V_{+} / V .
$$

If $V_{+} / V$ depended on one parameter it was slowly varying with that parameter, especially in those parameter regions that are usually of interest for the insurer. In these cases and for all other underlying distribution functions where $V+/ V$ is almost invariant for parameter changes in certain regions we can replace in these regions with a good approximation the semivariance as a risk measure by the variance.

The advantageous properties of $V+$ are then approximately (AI.) or generally (A3., A4.) fulfilled by $V$.

On the other hand we can indeed conclude that the "theoretical" properties of $V$ are also generally approximately fulfilled by $V_{+}$ (DI., D4.) but the "practical" disadvantages of $V_{+}$as compared to $V\left(\mathrm{D}_{2} ., \mathrm{D}_{3}\right.$.) are neither removed nor facilitated.

We therefore conclude that the variance is usually to be preferred to the semivariance as a risk measure. However we do not exclude the possibility that for special cases the semivariance may be preferable to the variance.

\section{REFERENCES}

[I] Beard, R. E., T. I'entikajnen, and E. Pesonen, Risk Theory, Methuen \& Co Ltd., I969, P. 4I, 42. 
[2] Benktander, G., The Calculation of a Fluctuation Loading for an Excess of Loss Cover, presented at ASTIN Colloquium in Turku, 1974, to be published.

[3] Benktander, G., On the Rating of a Special Stop Loss Cover, presented at ASTIN Colloquium in Turku, I974, to be published.

[4] Benktander, G., Some Aspects on Reinsurance Profits and Loadings, ASTIN Bulletin Vol. V, Part 3.

[5] Benktander, G., A Note on Profit Margin and Insurance Market Capacity, Mitteilungen der Vereinigung Schweizerischer Versicherungsmathematiker, Vol. 70, Nr. I, 1970.

[6] Berliner, B., On the Choice of Risk Loadings, to be presented at the 2oth International Congress of Actuaries in Tokyo, 1976.

17] Berliner, B., Some Thoughts on (Re)insurance Loadings under a Ruin Critcrion, Scandinavian Actuarial Journal, 1974, p. 78.

[8] Bowers Jr., N. L., An Upper Bound on the Stop Loss Net l'remium, Society of Actuaries Transactions, Vol. XXI, Part I, 1969.

[9] Gerber, H., On Additive Premium Calculation Principles, ASTIN Bulletin Vol. VII, Part 3.

[10] Markowitz, H., Portfolio Selection, Cowles Foundation for Research in Economics at Yale University, 1959, p. I88-201.

[i1] Schneewziss, H., Entscheidungskriterien bei Risiko, Springer-Verlag, 1967, p. 57 .

[12] VAN DeR WAERden, B. L., Mathematische Statistik, Springer-Verlag. I 965,2 . Auflage, p. 57. 